\title{
Risk Factors for Problem Drinking among Evacuees in Fukushima following the Great East Japan Earthquake: The Fukushima Health Management Survey
}

\author{
Yuka Ueda, ${ }^{1,2}$ Michio Murakami, ${ }^{3,4}$ Masaharu Maeda, ${ }^{4,5}$ Hirooki Yabe, ${ }^{1,4}$ \\ Yuriko Suzuki, ${ }^{6,7}$ Masatsugu Orui, ${ }^{7}$ Seiji Yasumura ${ }^{4,7}$ and Tetsuya Ohira; ${ }^{2,4}$ \\ The Fukushima Health Management Survey Group \\ ${ }^{1}$ Department of Neuropsychiatry, Fukushima Medical University School of Medicine, Fukushima, Fukushima, \\ Japan \\ ${ }^{2}$ Department of Epidemiology, Fukushima Medical University School of Medicine, Fukushima, Fukushima, Japan \\ ${ }^{3}$ Department of Health Risk Communication, Fukushima Medical University School of Medicine, Fukushima, \\ Fukushima, Japan \\ ${ }^{4}$ Radiation Medical Science Center for the Fukushima Health Management Survey, Fukushima Medical \\ University, Fukushima, Fukushima, Japan \\ ${ }^{5}$ Department of Disaster Psychiatry, Fukushima Medical University School of Medicine, Fukushima, Fukushima, \\ Japan \\ ${ }^{6}$ Department of Mental Health Policy, National Institute of Mental Health, National Center of Neurology and \\ Psychiatry, Tokyo, Japan \\ ${ }^{7}$ Department of Public Health, Fukushima Medical University School of Medicine, Fukushima, Japan
}

\begin{abstract}
Many studies have consistently reported the bidirectional relationship between problem drinking and psychological distress following a disaster, but the risk factors of problem drinking following a disaster remain unclear. In this study, we therefore aimed to explore the risk factors associated with the incidence of problem drinking among evacuees after the Great East Japan Earthquake of March 11, 2011. We used the data for evacuees of the Fukushima Daiichi nuclear power plant accident, obtained from the Mental Health and Lifestyle Survey. A total of 12,490 individuals from 13 municipalities, which included the evacuation order areas after the accident, completed surveys between 2012 and 2013. The CAGE (Cutting down, Annoyed by criticism, Guilty feeling, and Eye-opener) questionnaire was used to screen the participants for alcohol dependence, and a score $\geq 2$ indicated problem drinking. Logistic regression models were applied to investigate the possible predictors of problem drinking. The results showed that insufficient sleep and heavy drinking ( $\geq 4$ drinks per day) were significant risk factors for the incidence of problem drinking in both men and women. Additional risk factors included family financial issues due to the disaster and trauma symptoms among men and a diagnosed history of mental illness among women. Other remaining variables were not significantly associated with problem drinking. The present study is the first to identify the risk factors for problem drinking following a compound disaster. Our findings could be used to develop a primary intervention program to improve evacuees' health and lives following a disaster.
\end{abstract}

Keywords: cutting down, annoyed by criticism, guilty feeling, and eye-opener (CAGE); disaster; epidemiology; evacuees; problem drinking

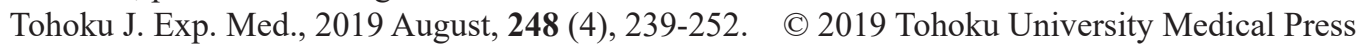

\section{Introduction}

Alcohol and mental health are interrelated and known to influence each other (Bell et al. 2014). Verplaetse et al. (2018) explained that substance use disorders are often associated with stressful events, and it is common for individuals with an alcohol use disorder to have comorbid posttraumatic stress disorder (PTSD) or past experience of trau- matic events (Petrakis and Simpson 2017). Particularly, individuals who experience a disaster tend to be at risk for increased alcohol consumption (Fullerton et al. 2013; Welch et al. 2014). In the first and second years after the $9 / 11$ terrorist attacks, alcohol consumption, binge drinking, and alcohol misuse were associated with posttraumatic stress disorder onset (Boscarino et al. 2011).

The Great East Japan Earthquake of March 11, 2011

Received March 13, 2019; revised and accepted July 24, 2019. Published online August 10, 2019; doi: 10.1620/tjem.248.239.

Correspondence: Yuka Ueda, M.A., Department of Neuropsychiatry, Fukushima Medical University (FMU), 1 Hikarigaoka, Fukushima,

Fukushima 960-1295, Japan.

e-mail: yumu327@fmu.ac.jp 
and the subsequent Fukushima nuclear accident constituted a compound disaster that caused problem drinking and psychological stress among evacuees from Fukushima (Oe et al. 2016). In our previous study on Fukushima evacuees, those who started drinking after the disaster showed a higher risk of serious mental illness after the disaster (Ueda et al. 2016).

Attention and intervention for alcohol-related issues are essential after disasters. However, the risk factors underlying the problem drinking of disaster evacuees remain unclear. Understanding these factors would help us support evacuees trying to reconstruct their lives, and enable the integration of alcohol-misuse prevention programs into global disaster preparation and response efforts. This prospective study, therefore, assessed how trauma exposure and various psychological and social risk factors influenced problem drinking among evacuees of the Great East Japan Earthquake. Previous studies have linked demographic characteristics, socioeconomic factors, and disaster-related factors with problem drinking (Boscarino et al. 2006, 2011; Gray et al. 2016). Therefore, our a priori hypothesis is that family financial problems and employment changes due to disaster, as well as physical and psychological problems including sleep insufficiency, will be predictors of problem drinking among evacuees.

\section{Methods}

\section{Study design}

We used data from the Mental Health and Lifestyle Survey, which assessed the mental health and lifestyle of evacuees of the Fukushima Daiichi nuclear power plant accident. The complete survey protocol was published in 2012 (Yasumura et al. 2012). Target participants lived in the 12 municipalities that were issued evacuation orders by the government at the time of the accident (Hirono, Naraha, Tomioka, Kwauchi, Okuma, Futaba, Namie, Katsurao, Iitate, Tamura, Minami-Soma, and Kawamata) and in evacuated hot-spot areas in Date. While all residents of Hirono, Naraha, Tomioka, Kwauchi, Okuma, Futaba, Namie, Katsurao, and Iitate were ordered to evacuate, the municipalities of Minami-Soma, Tamura, and Kawamata included both evacuees and non-evacuees. These residents have received questionnaires yearly since January 18, 2012 (Yasumura et al. 2012). This paper used data only from 2012 and 2013 to elucidate the development of problem drinking in the first two years post-disaster. The Ethics Review Committee of Fukushima Medical University approved this study (No. 1316).

\section{Participants}

Fig. 1 shows the participant flow chart. The target population was 52,602 adults aged 20 years old or older who responded to the 2012 survey (response rate $19.9 \% ; \mathrm{n}=184,507$ ). We excluded respondents who failed to answer the CAGE (Cut down, Annoyed, Guilty, and Eye-opener) questionnaire $(\mathrm{n}=12,247)$ or who did not respond to the questionnaire on their own $(n=3,662)$ for 2012 . Then, we again excluded respondents who failed to answer the CAGE questionnaire $(n=9,805)$ and who did not respond on their own $(n=$ 479) for 2013. Participants reporting a CAGE score $<2$ accounted for $95.3 \%$ of the sample $(n=9,802)$, while $482(4.7 \%)$ participants

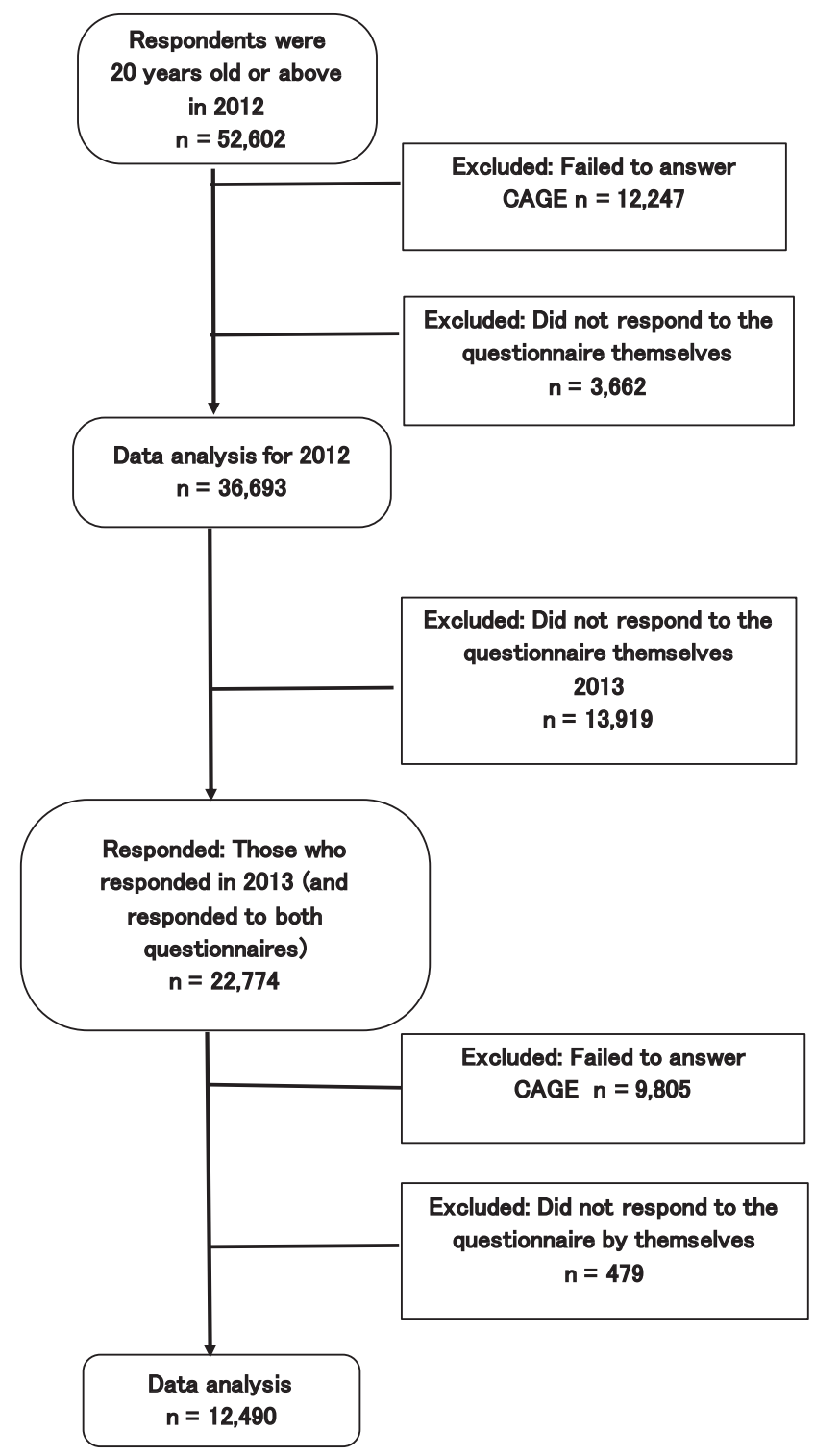

Fig. 1. Participant flow chart.

who reported a CAGE score $\geq 2$. Therefore, we ultimately obtained longitudinal data for 12,490 participants.

Measures

Alcohol consumption and problem drinking. Respondents rated their current drinking behavior as "don't drink or only rarely drink (less than once a month)," "quit drinking," or "drink (one or more times a month)." If they responded, "drink (one or more times a month)," they were asked to specify how much they drank in a year. To be consistent with our previous research (Ueda et al. 2016), heavy drinking/drinking consumption that would enhance the risk for a lifestyle disease was defined as four drinks per day ( $\geq 44 \mathrm{~g}$ of ethanol); lower consumption levels were defined as "light drinking." A drink, by this definition, meant $120 \mathrm{~mL}$ of spirits (e.g., whisky or brandy), $480 \mathrm{~mL}$ of wine, $1,000 \mathrm{~mL}$ of beer, or $360 \mathrm{~mL}$ of sake. This definition is consistent with the reported median of moderate drinking ( $20 \mathrm{~g}$ of ethanol per day) and heavy drinking (60 g of ethanol per day) reported by the Japanese Ministry of Health, Labour and Welfare (2019). 
The CAGE questionnaire was administered only to those with the responses "don't drink or only rarely drink (less than once a month)" and "drink (one or more times a month)" to assess whether they were problem drinkers. The CAGE questionnaire was designed to screen for alcohol dependency (Castells and Furlanetto 2005), and its validity and effectiveness have been confirmed by past research. It can also aid in the diagnosis of alcoholism (Ewing 1984). It comprises four questions: 1) "Have you ever felt you ought to Cut down on your drinking," 2) "Have people Annoyed you by criticizing your drinking," 3) "Have you ever felt bad or Guilty about your drinking," and 4) "Have you ever had a drink first thing in the morning to steady your nerves or to get rid of a hangover (Eye-opener)?" (Ewing 1984). Participants with two or more positive answers were classified as having alcohol dependence irrespective of their sex in accordance with the previous studies (Castells and Furlanetto 2005). For the purpose of this research, we defined a CAGE score of $\geq 2$ as problem drinking. We also categorized participants according to whether they experienced changes in CAGE score from 2012 to 2013 as "emerging problem drinkers," comprising individuals without alcohol dependence in 2012, but with alcohol dependence (i.e., incidence of problem drinking) in 2013, and "maintaining non-problem drinkers," comprising individuals who demonstrated alcohol dependence in neither 2012 nor 2013.

General and socioeconomic status variables: Previous studies have linked various demographic characteristics, socioeconomic factors, and disaster-related factors with problem drinking (Boscarino et al. 2006, 2011; Gray et al. 2016). Demographic factors considered here included sex, age (20-49, 50-64, or $\geq 65$ years), and any history of a diagnosed mental illness (yes or no), high blood pressure (yes or no), diabetes mellitus (yes or no), or hyperlipidemia (yes or no). Socioeconomic factors included employment change (i.e., change in work from pre- to post-disaster; yes or no) and family financial situation post-disaster (severe, somewhat severe, average, or not severe).

Current social network status: To assess current social network status, we used the Lubben Social Network Scale (LSNS-6). This scale assesses family and friendship ties and has been empirically validated for screening for risk of social isolation (Lubben et al. 2006; Nordløkken et al. 2016). The scale was constructed from three items for each of the two ties, as follows: 1) "How many relatives do you see or hear from at least once a month," 2) "How many relatives do you feel close to such that you could call on them for help," 3 ) "How many relatives do you feel at ease with that you can talk about private matters," 4) "How many of your friends do you see or hear from at least once a month," 5) "How many friends do you feel close to such that you could call on them for help," and 6) "How many friends do you feel at ease with that you can talk about private matters" (Nordløkken et al. 2016). Each item is rated on a 6-point scale: 0 (none), 1 (one), 2 (two), 3 (three or four), 4 (five to eight), and 5 (nine or more). We classified participants with scores of $<12 / 30$ points as being at risk of social isolation (Lubben et al. 2006). The Japanese version of the LSNS-6 has shown adequate reliability and validity (Kurimoto et al. 2011).

Sleep insufficiency: Traumatic events also lead to sleep-related problems (Lavie 2001). Sleep disruption was one of the most frequently cited issues after the Great East Japan Earthquake (Matsumoto et al. 2014). Alcohol is also often used as a (poor) coping mechanism for insomnia (Karz et al. 2014). In the questionnaire, we asked, "Are you satisfied with the quality of your sleep over the past month (regardless of sleep duration)?" Participants indicated if they were "satisfied," "slightly dissatisfied," "quite dissatisfied," or "very dissatisfied or have barely slept at all."

Laughter. We also analyzed the frequency of laughter. Laughter enhances individual's emotional well-being and life satisfaction (Hasan and Hasan 2009). The participants of this study were asked how often they had laughed in the previous month: "every day," "1-5 times per week," "1-3 times per month," or "none." Greater frequency of laughter can promote the general and mental health of older adults in Japan (Hayashi et al. 2015). This question has proven to have adequate test-retest reliability (Hirosaki et al. 2018).

Risk of serious mental illness and psychological distress (K6): We used the 6-item Kessler Psychological Distress Scale (K6) as a screener for non-specific serious mental illness (Kessler et al. 2003). Scores of 13-24 were classified as "probable serious mental illness," while scores of 0-12 were classified as "probable mild-moderate/ probable no mental illness" (Kessler et al. 2006). The current study used the Japanese version of the K6, which was validated in a previous study (Furukawa et al. 2008).

Trauma symptoms: We used the PTSD Checklist-Specific (PCLS) to measure current traumatic symptoms among participants, to analyze how they were related to drinking behavior. The PCL-S comprises 17 items assessing PTSD symptoms, all of which are rated on a Likert scale from 1 (not at all) to 5 (extremely). A cutoff of 44 was able to correctly identify individuals with a PTSD diagnosis (Blanchard et al. 1996). We used the Japanese version of the PCL-S, which was previously validated (Iwasa et al. 2016; Suzuki et al. 2017).

\section{Statistical analysis}

First, we performed a chi-squared test to investigate associations between risk factors and the proportion of individuals who were emerging problem drinkers. The data were then analyzed only for those who did not have a drinking problem (CAGE < 2) in 2012. Bivariate logistic regression models were applied to investigate the possible predictors of problem drinking development (i.e., emerging problem drinkers). We further conducted a multivariate logistic regression analysis that included the significant variables from the bivariate analysis. We excluded missing data from the statistical analysis.

Furthermore, to confirm the representativeness of the participants, we performed a chi-squared test to compare sociodemographic, health-related, and disaster-related status in 2012 between those who completed the CAGE in $2012(\mathrm{n}=36,693)$ and those who responded in 2012, excluding participants with missing CAGE data or failure to respond $(n=15,909)$. Further, we performed a bivariate logistic regression model to compare the odds ratios of individual risk factors for the prevalence of problem drinking in 2012 between the participants analyzed in this study $(\mathrm{n}=12,490)$, and those who were excluded because they responded only in 2012, but not $2013(\mathrm{n}=13,946)$.

Significance was set at $p<.05$. All statistical analyses were conducted using IBM SPSS Statistics 24.0 (IBM Corp. Armonk, NY).

\section{Results}

Table 1 shows the breakdown of variables according to change (or lack thereof) in problem drinking (low score to high score defined as emerging problem drinkers; 
continuous low score defined as maintaining non-problem drinkers) from 2012 to 2013, revealing what characteristics were associated with these changes. The total number of emerging problem drinkers was 902 , while the number of maintaining non-problem drinkers was 9,693. Importantly, $8.5 \%$ of the sample developed problem drinking from 2012 to 2013 , the year following the disaster.

More men than women developed problem drinking from 2012 to 2013. Emerging problem drinkers also included a higher proportion of those with K6, PCL-S, and LSN-6 scores of $\geq 13, \geq 44$, and $<12$, respectively, and were heavy drinkers compared to maintaining non-problem drinkers. Furthermore, we found becoming a problem drinker to be associated with age, subjective health, history of a serious mental illness, high blood pressure, diabetes mellitus, sleep insufficiency, laughter frequency, employment change, and family financial status $(p<.05)$.

Table 2 shows an overview of the bivariate logistic regression analysis of both emerging problem drinkers and maintaining non-problematic drinkers, conducted to identify possible predictors for developing problem drinking from 2012 to 2013. None of the variance inflation factors exceeded two, indicating no collinearity. We found that the development of problem drinking was significantly predicted by sex, subjective health conditions, diagnosed mental illness history, blood pressure, diabetes mellitus, sleep insufficiency, laughing frequency, employment change due to disaster, family finances, alcohol consumption, and specific scores on the K6, PCL-S, and LSN-6.

In the multivariate logistic regression analysis (Table 3 ), sex (odds ratio $[\mathrm{OR}]=1.77,95 \% \mathrm{CI}$ : $1.44-2.21$ ), age (20-49 years old) (odds ratio $[\mathrm{OR}]=1.38,95 \% \mathrm{CI}$ : 1.04 1.82), sleep insufficiency (OR $=1.63,95 \% \mathrm{CI}: 1.21-2.19)$, PCL-S $\geq 44$ (OR $=1.75,95 \%$ CI: 1.33-2.31), family finances $([$ severe] $\mathrm{OR}=1.71,95 \% \mathrm{CI}$ : 1.33-2.20; [not severe] $\mathrm{OR}=0.22,95 \% \mathrm{CI}$ : $0.08-0.59)$ and heavy drinking $(\mathrm{OR}=2.26,95 \% \mathrm{CI}: 1.82-2.80)$ remained significantly associated with the development of problem drinking. There were also some differences in the predictors by sex. Among men, age $(\mathrm{OR}=1.29$ 95\%CI: 1.00-1.66), family finances ([severe] $\mathrm{OR}=1.81,95 \% \mathrm{CI}: 1.36-2.42$; [not severe] $\mathrm{OR}=0.15,95 \% \mathrm{CI}: 0.04-0.60)$, and $\mathrm{PCL} \geq 44(\mathrm{OR}=2.08$, 95\% CI: 1.52-2.84) were also risk factors for problem drinking. Among women, history of a diagnosed mental illness $(\mathrm{OR}=1.99,95 \%$ CI: $1.06-3.74)$ was a significant risk factor.

As there were particularly strong correlations between the K6 and PCL-S, we conducted separate multivariate logistic regression analyses for these predictors to identify their independent predictive ability for problem drinking development (Tables 4 and 5). While there were no significant associations between $\mathrm{K} 6$ and the development of problem drinking from 2012 to 2013 (Table 4), PCL-S was a risk factor for both men and women (Table 5).

\section{Discussion}

This study examined the risk factors for developing problem drinking from 2012 to 2013, after the Great East Japan Earthquake, among Fukushima evacuees. North et al. (2011) suggested that the clinically significant risk factors for problem drinking have not been systematically evaluated, and that the development of problem drinking following disasters need to be considered in terms of both pathological and social factors. Additionally, a study conducted in the second year after this compound disaster showed that the risk factors for developing post-disaster problem drinking included being male or less than 65 years, having sleep insufficiency or psychological distress, and drinking heavily (Orui et al. 2017).

In this study, a sizable proportion of the sample $(8.5 \%)$ developed problem drinking within a year (from 2012 to 2013) of the compound disaster. These results highlight the importance of developing primary prevention plans in public health for alcohol problems during the chronic phase post-disaster. We hypothesized that disaster-related factors such as employment changes due to disasters and physical and psychological problems are associated with an increased risk of developing problem drinking from the second to the third year post-disaster.

According to our prospective analysis, sleep insufficiency and heavy drinking were major predictors of developing problem drinking from 2012 to 2013 in both sexes. Additionally, we observed some differences between the sexes in the predictors of problem-drinking development. Among men, having trauma symptoms (PCL-S $\geq 44$ ) and a severe change in family finances due to the disaster were significant risk factors for problemdrinking development; among women, mental illness history was a significant predictor. It is important to consider such differences in risk factors between sexes when devising intervention plans for problem drinking.

Our findings counter those of some previous studies that investigated the risk factors for alcohol problems in the post-disaster period (Cerda et al. 2011; Kachadourian et al. 2014). Several previous studies found that a low-income trajectory was a factor for changing alcohol consumption (Vlahov et al. 2002; Cerda et al. 2008, 2011). Additionally, having low income post-disaster has been associated with depression (Ahern and Galea 2006). In this study, however, we found that family financial situation was a significant predictor only for men. Additionally, a previous national study found that trauma exposure/PTSD was more strongly associated with problem drinking in women compared to men (Kachadourian et al. 2014). This contrasts with our result that trauma influenced problem drinking only in men. However, our results support, to a certain extent, a study stating that women show increased use of pharmaceuticals after a disaster, while men show increased use of alcohol (Vetter et al. 2008). Additionally, Boscarino et al. (2011) examined the experiences of the 9/11 terrorist attack vic- 
Table 1. Relationships among possible risk factors for problem drinking according to increases in CAGE scores.

\begin{tabular}{|c|c|c|c|c|c|c|c|c|}
\hline & Total & $\begin{array}{l}\text { Emerging problem } \\
\text { drinkers } \\
C A G E<2 \text { in } 2012 \rightarrow \\
C A G E \geq 2 \text { in } 2013\end{array}$ & $\%$ & $\begin{array}{l}\text { Maintaining non-problem } \\
\text { drinkers } \\
\text { CAGE }<2 \text { in } 2012 \rightarrow \\
\text { CAGE }<2 \text { in } 2013\end{array}$ & $\%$ & df & $\chi^{2}$ & $p$ \\
\hline Sex & 10,595 & & & & & \multirow{3}{*}{1} & \multirow{3}{*}{141} & \multirow{3}{*}{$<0.001$} \\
\hline Male & 6,264 & 701 & 77.7 & 5,563 & 57.4 & & & \\
\hline Female & 4,331 & 201 & 22.3 & 4,130 & 42.6 & & & \\
\hline Age & 10,595 & & & & & & & \\
\hline 20-49 years old & 3,054 & 227 & 25.2 & 2,827 & 29.2 & \multirow{3}{*}{2} & \multirow{3}{*}{6.4} & \multirow{3}{*}{0.04} \\
\hline 50-64 years old & 3,758 & 335 & 37.1 & 3,423 & 35.3 & & & \\
\hline$\geq 65$ years old & 3,783 & 340 & 37.7 & 3,443 & 35.5 & & & \\
\hline Subjective health condition & 10,353 & & & & & \multirow{4}{*}{2} & \multirow{4}{*}{32.4} & \multirow{4}{*}{$<0.001$} \\
\hline Very good-Good & 2,374 & 153 & 17.3 & 2,221 & 23.5 & & & \\
\hline Normal & 6,521 & 558 & 63.3 & 5,963 & 63 & & & \\
\hline Poor-Very poor & 1,458 & 171 & 19.4 & 1,287 & 13.6 & & & \\
\hline Diagnosed history of mental illness & 10,425 & & & & & \multirow{3}{*}{1} & \multirow{3}{*}{17} & \multirow{3}{*}{$<0.00$} \\
\hline No & 9,790 & 803 & 90.7 & 8,987 & 94.2 & & & \\
\hline Yes & 635 & 82 & 9.3 & 553 & 5.8 & & & \\
\hline Diagnosed with high blood pressure & 10,504 & & & & & \multirow{3}{*}{1} & \multirow{3}{*}{33.9} & \\
\hline No & 5,780 & 406 & 45.7 & 5,374 & 55.9 & & & \\
\hline Yes & 4,724 & 482 & 54.3 & 4,242 & 44.1 & & & $<0.001$ \\
\hline Diagnosed with diabetes mellitus & 10,429 & & & & & \multirow{3}{*}{1} & \multirow{3}{*}{15.8} & \\
\hline No & 8,375 & 661 & 75.2 & 7,714 & 80.8 & & & $<0.001$ \\
\hline Yes & 2,054 & 218 & 24.8 & 1,836 & 19.2 & & & \\
\hline Diagnosed with hyperlipidemia & 10,430 & & & & & & & \\
\hline No & 6,322 & 514 & 58.7 & 5,808 & 60.8 & 1 & 1.4 & 0.24 \\
\hline Yes & 4,108 & 361 & 41.3 & 3,747 & 39.2 & & & \\
\hline Exercise & 10,457 & & & & & & & \\
\hline Every day & 1,505 & 143 & 16.2 & 1,362 & 14.2 & & & \\
\hline 2-4 times a week & 2,397 & 208 & 23.5 & 2,189 & 22.9 & 3 & 46 & 021 \\
\hline Once a week & 1,705 & 127 & 14.4 & 1,578 & 16.5 & 3 & 4.0 & 0.21 \\
\hline None & 4,850 & 406 & 45.9 & 4,444 & 46.4 & & & \\
\hline Sleep insufficiency & 10,310 & & & & & & & \\
\hline Satisfied & 4,030 & 289 & 33 & 3,741 & 39.7 & & & \\
\hline A little dissatisfied & 4,711 & 403 & 46.1 & 4,308 & 45.7 & 2 & 29.4 & $<0.001$ \\
\hline Very dissatisfied to quite problematic & 1,569 & 183 & 20.9 & 1,386 & 14.7 & & & \\
\hline Laughing frequency & 10,529 & & & & & & & \\
\hline Almost every day & 2,845 & 198 & 22.1 & 2,647 & 27.5 & & & \\
\hline $1-5$ days per week & 4,424 & 373 & 41.6 & 4,051 & 42.1 & 3 & 195 & $<0,001$ \\
\hline $1-3$ days per month & 2,070 & 198 & 22.1 & 1,872 & 19.4 & & & \\
\hline Almost never & 1,190 & 128 & 14.3 & 1,062 & 11 & & & \\
\hline Employment change & 9,732 & & & & & & & \\
\hline Yes & 5,199 & 491 & 60 & 4,708 & 52.8 & 1 & 153 & $<0.001$ \\
\hline No & 4,533 & 328 & 40 & 4,205 & 47.2 & 1 & & \\
\hline Family finances & 10,041 & & & & & & & \\
\hline Severe & 1,574 & 222 & 26.2 & 1,352 & 14.7 & & & \\
\hline Below average & 2,996 & 277 & 32.7 & 2,719 & 29.6 & 3 & 1109 & $<0001$ \\
\hline Average & 5,085 & 342 & 40.4 & $4,743.00$ & 51.6 & & & \\
\hline Not severe & 386 & 6 & 0.7 & 380 & 4.1 & & & \\
\hline Psychological distress & 10,251 & & & & & & & \\
\hline K $6<13$ & 9,412 & 747 & 87 & 8,665 & 92.3 & 1 & 294 & $<0.001$ \\
\hline$K 6 \geq 13$ & 839 & 112 & 13 & 727 & 7.7 & 1 & & \\
\hline Trauma symptoms & 9,778 & & & & & & & \\
\hline $\mathrm{PCL}<44$ & 8,466 & 621 & 75.3 & 7,845 & 87.6 & 1 & 99.2 & $<0.001$ \\
\hline $\mathrm{PCL} \geq 44$ & 1,312 & 204 & 24.7 & 1,108 & 12.4 & 1 & 99.2 & $<0.001$ \\
\hline Social network & 10,268 & & & & & & & \\
\hline LSN_6 $<12$ & 3,874 & 356 & 41.1 & 3,518 & 37.4 & 1 & 45 & $<0.001$ \\
\hline LSN_6 $\geq 12$ & 6,394 & 511 & 58.9 & 5,883 & 62.6 & 1 & 4.5 & \\
\hline Alcohol consumption (drinks) & 8,074 & & & & & & & \\
\hline$<4$ & 6,917 & 553 & 72.4 & 6,364 & 87.1 & 1 & 1214 & $<0,001$ \\
\hline$\geq 4$ & 1,157 & 211 & 27.6 & 946 & 12.9 & 1 & 121.4 & $<0.001$ \\
\hline
\end{tabular}

CAGE, cutting down, annoyed by criticism, guilty feeling, and eye-opener questionnaire; LSN, Lubben social network scale; PCL, PTSD checklist-specific; K6, Kessler psychological distress scale. 
Y. Ueda et al.

Table 2. Bivariate analysis: possible risk factors for problem drinking according to increase in CAGE score.

\begin{tabular}{lccccccc}
\hline & \multicolumn{2}{c}{ All } & \multicolumn{2}{c}{ Male } & \multicolumn{2}{c}{ Female } \\
& OR & $95 \%$ CI & OR & $95 \%$ CI & OR & $95 \%$ CI \\
\hline Sex (Reference: Female) & & & & & & & \\
Male & 2.64 & $2.24-3.12$ & & & & \\
& & & & & & & \\
Age (Reference: $\geq 65$ years old) & & & & & & & \\
$20-49$ years old & 1.08 & $0.90-1.29$ & 0.92 & $0.74-1.14$ & 1.62 & $1.08-2.42$ \\
$50-64$ years old & 1.15 & $0.98-1.35$ & 1.16 & $0.97-1.38$ & 1.27 & $0.83-1.94$ \\
& & & & & & & \\
Subjective health condition (Reference: Very good-Good) & & & & & & \\
Normal & 1.44 & $1.19-1.73$ & 1.34 & $1.09-1.65$ & 1.94 & $1.25-3.03$ \\
Poor-Very poor & 2.05 & $1.62-2.58$ & 1.92 & $1.48-2.49$ & 2.73 & $1.62-4.62$ \\
& & & & & & & \\
Exercise (Reference: Every day) & & & & & & \\
$2-4$ times a week & 0.97 & $0.78-1.22$ & 1.01 & $0.79-1.29$ & 0.86 & $0.47-1.55$ \\
Once a week & 0.84 & $0.65-1.08$ & 0.79 & $0.60-1.06$ & 1.02 & $0.56-1.86$ \\
None & 0.98 & $0.79-1.21$ & 0.97 & $0.77-1.23$ & 0.99 & $0.58-1.71$
\end{tabular}

Diagnosed history of mental illness (Reference: No)

Yes

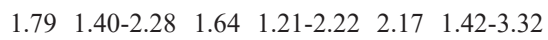

Diagnosed with high blood pressure (Reference: None)

Yes

$\begin{array}{llllll}1.38 & 1.17-1.61 & 1.39 & 1.16-1.66 & 1.34 & 0.94-1.90\end{array}$

Diagnosed with diabetes mellitus (Reference: No)

Yes

$\begin{array}{llllll}1.26 & 1.06-1.49 & 1.29 & 1.07-1.55 & 1.08 & 0.68-1.71\end{array}$

Diagnosed with hyperlipidemia (Reference: No)

Yes

$\begin{array}{lllllll}1.02 & 0.89-1.19 & 1.08 & 0.92-1.27 & 0.90 & 0.64-1.26\end{array}$

Sleep insufficiency (Reference: Satisfied)

A little dissatisfied

Very dissatisfied to quite problematic

$\begin{array}{lllllll}1.33 & 1.13-1.56 & 1.30 & 1.09-1.56 & 1.51 & 1.05-2.18\end{array}$

$\begin{array}{llllll}1.94 & 1.59-2.37 & 1.86 & 1.47-2.35 & 2.34 & 1.56-3.55\end{array}$

Laughing frequency (Reference: Almost every day)

$1-5$ days per week

1-3 days per month

$\begin{array}{llllll}1.17 & 0.98-1.40 & 1.15 & 0.93-1.42 & 1.21 & 0.85-1.74\end{array}$

$\begin{array}{llllll}1.27 & 1.03-1.56 & 1.15 & 0.90-1.46 & 1.75 & 1.15-2.65\end{array}$

Almost never

$\begin{array}{llllll}1.38 & 1.08-1.74 & 1.23 & 0.94-1.61 & 2.13 & 1.29-3.51\end{array}$

Employment change (Reference: No)

Yes

$\begin{array}{llllll}1.37 & 1.18-1.59 & 1.42 & 1.19-1.68 & 1.22 & 0.90-1.65\end{array}$

Family finances (Reference: Average)

Severe

Below average

Not severe

$\begin{array}{llllll}2.19 & 1.83-2.63 & 2.38 & 1.94-2.92 & 1.67 & 1.13-2.48\end{array}$

$\begin{array}{llllll}1.36 & 1.15-1.61 & 1.31 & 1.08-1.59 & 1.51 & 1.09-2.10\end{array}$

$\begin{array}{lllllll}0.22 & 0.10-0.49 & 0.1 & 0.02-0.39 & 0.60 & 0.22-1.65\end{array}$

Psychological distress (Reference: K6 $<13$ )

$\mathrm{K} 6 \geq 13$

$\begin{array}{llllll}1.96 & 1.58-2.44 & 1.89 & 1.45-2.45 & 2.18 & 1.49-3.18\end{array}$

Trauma symptom (Reference: PCL $<44$ )

$\mathrm{PCL} \geq 44$

$\begin{array}{llllll}2.51 & 2.11-3.00 & 2.66 & 2.18-3.25 & 2.16 & 1.51-3.08\end{array}$

Social network (Reference: LSN_6 $\geq 12$ )

LSN $6<12$

$\begin{array}{llllll}1.15 & 1.00-1.33 & 1.11 & 0.93-1.31 & 1.33 & 1.00-1.78\end{array}$

Alcohol consumption (Reference: $<4$ drinks)

$\geq 4$ drinks

\begin{tabular}{llllll}
2.21 & $1.85-2.64$ & 2.01 & $1.67-2.43$ & 4.41 & $2.71-7.19$ \\
\hline
\end{tabular}

CAGE, cutting down, annoyed by criticism, guilty feeling, and eye-opener questionnaire;

LSN, Lubben social network scale; PCL, PTSD checklist-specific; K6, Kessler psycho-

logical distress scale. 
Table 3. Multivariate analysis: possible risk factors for problem drinking according to increase in CAGE score.

\begin{tabular}{|c|c|c|c|c|c|c|}
\hline & & All & & Male & & emale \\
\hline & OR & $95 \% \mathrm{CI}$ & OR & $95 \% \mathrm{CI}$ & OR & $95 \% \mathrm{CI}$ \\
\hline Sex (Reference: Female) & & & & & & \\
\hline Male & 1.77 & $1.41-2.21$ & & & & \\
\hline Age (Reference: $\geq 65$ years old) & & & & & & \\
\hline $20-49$ years old & 1.38 & $1.04-1.82$ & 1.25 & $0.91-1.72$ & 1.88 & $0.93-3.80$ \\
\hline 50-64 years old & 1.25 & $0.98-1.58$ & 1.29 & $1.00-1.66$ & 1.27 & $0.64-2.53$ \\
\hline Subjective health condition (Referenc & od) & & & & & \\
\hline Normal & 1.19 & $0.93-1.52$ & 1.20 & $0.91-1.58$ & 1.12 & $0.66-1.91$ \\
\hline Poor-Very poor & 1.04 & $0.73-1.48$ & 1.10 & $0.73-1.65$ & 0.86 & $0.41-1.81$ \\
\hline Diagnosed history of mental illness ( $\mathrm{R}$ & & & & & & \\
\hline Yes & 1.27 & $0.87-1.84$ & 1.02 & $0.64-1.63$ & 1.99 & $1.06-3.74$ \\
\hline Diagnosed with high blood pressure (I & & & & & & \\
\hline Yes & 1.13 & $0.91-1.40$ & 1.14 & $0.90-1.45$ & 1.03 & $0.63-1.70$ \\
\hline Diagnosed with diabetes mellitus (Ref & & & & & & \\
\hline Yes & 1.16 & $0.90-1.48$ & 1.18 & $0.90-1.53$ & 1.01 & $0.49-2.11$ \\
\hline Sleep insufficiency (Reference: Satisf & & & & & & \\
\hline A little dissatisfied & 1.34 & $1.08-1.66$ & 1.19 & $0.94-1.52$ & 2.35 & $1.36-4.05$ \\
\hline Very dissatisfied to quite problematic & 1.63 & $1.21-2.19$ & 1.41 & $1.00-2.00$ & 3.01 & $1.59-5.66$ \\
\hline Laughing frequency (Reference: Alm & & & & & & \\
\hline 1-5 days per week & 1.21 & $0.96-1.53$ & 1.19 & $0.90-1.57$ & 1.29 & $0.82-2.03$ \\
\hline $1-3$ days per month & 1.16 & $0.88-1.54$ & 1.09 & $0.78-1.50$ & 1.50 & $0.85-2.66$ \\
\hline Almost never & 0.89 & $0.62-1.26$ & 0.86 & $0.58-1.28$ & 0.95 & $0.42-2.17$ \\
\hline Employment change (Reference: No) & & & & & & \\
\hline Yes & 1.10 & $0.91-1.34$ & 1.16 & $0.93-1.44$ & 0.95 & $0.64-1.39$ \\
\hline Family finances (Reference: Average) & & & & & & \\
\hline Severe & 1.71 & $1.33-2.20$ & 1.81 & $1.36-2.42$ & 1.31 & $0.76-2.27$ \\
\hline Below average & 1.22 & $0.98-1.51$ & 1.18 & $0.92-1.51$ & 1.25 & $0.82-1.90$ \\
\hline Not severe & 0.22 & $0.08-0.59$ & 0.15 & $0.04-0.60$ & 0.42 & $0.10-1.76$ \\
\hline Psychological distress (Reference: K6 & & & & & & \\
\hline$K 6 \geq 13$ & 0.98 & $0.69-1.39$ & 0.87 & $0.60-1.33$ & 1.28 & $0.67-2.45$ \\
\hline Trauma (Reference: PCL < 44) & & & & & & \\
\hline $\mathrm{PCL} \geq 44$ & 1.75 & $1.33-2.31$ & 2.08 & $1.52-2.84$ & 1.07 & $0.60-1.90$ \\
\hline Social network (Reference: LSN_6 $\geq$ & & & & & & \\
\hline LSN_6 $<12$ & 0.85 & $0.70-1.03$ & 0.87 & $0.70-1.10$ & 0.81 & $0.55-1.20$ \\
\hline Alcohol consumption (Reference: $<4$ & & & & & & \\
\hline$\geq 4$ drinks & 2.26 & $1.82-2.80$ & 2.12 & $1.69-2.66$ & 3.54 & $1.92-6.52$ \\
\hline
\end{tabular}

CAGE, cutting down, annoyed by criticism, guilty feeling, and eye-opener questionnaire; LSN, Lubben social network scale; PCL, PTSD checklist-specific; K6, Kessler psychological distress scale. 
Table 4. Multivariate analysis: possible risk factors for problem drinking according to increase in CAGE score (K6 only).

\begin{tabular}{|c|c|c|c|c|c|c|}
\hline & & All & & Male & & emale \\
\hline & OR & $95 \% \mathrm{CI}$ & OR & $95 \% \mathrm{CI}$ & OR & $95 \% \mathrm{CI}$ \\
\hline Sex (Reference: Female) & & & & & & \\
\hline Male & 1.66 & $1.34-2.06$ & & & & \\
\hline Age (Reference: $\geq 65$ years old) & & & & & & \\
\hline 20-49 years old & 1.29 & $0.99-1.69$ & 1.17 & $0.86-1.59$ & 1.86 & $0.98-3.52$ \\
\hline $50-64$ years old & 1.18 & $0.94-1.48$ & 1.20 & $0.93-1.52$ & 1.31 & $0.71-2.43$ \\
\hline Subjective health condition (Reference: & od) & & & & & \\
\hline Normal & 1.21 & $0.96-1.53$ & 1.21 & $0.93-1.58$ & 1.17 & $0.70-1.96$ \\
\hline Poor-Very poor & 1.16 & $0.83-1.62$ & 1.23 & $0.84-1.81$ & 0.97 & $0.48-1.96$ \\
\hline Diagnosed history of mental illness (Refe & & & & & & \\
\hline Yes & 1.31 & $0.91-1.86$ & 1.06 & $0.68-1.66$ & 2.05 & $1.13-3.72$ \\
\hline Diagnosed with high blood pressure (Ref & & & & & & \\
\hline Yes & 1.18 & $0.96-1.45$ & 1.21 & $0.96-1.53$ & 1.07 & $0.68-1.70$ \\
\hline Diagnosed with diabetes mellitus (Refere & & & & & & \\
\hline Yes & 1.14 & $0.90-1.44$ & 1.16 & $0.90-1.49$ & 1.03 & $0.52-2.02$ \\
\hline Sleep insufficiency (Reference: Satisfied & & & & & & \\
\hline A little dissatisfied & 1.25 & $1.01-1.53$ & 1.14 & $0.91-1.45$ & 1.91 & $1.17-3.14$ \\
\hline Very dissatisfied to quite problematic & 1.58 & $1.19-2.10$ & 1.43 & $1.02-2.00$ & 2.43 & $1.36-4.36$ \\
\hline Laughing frequency (Reference: Almost & & & & & & \\
\hline 1-5 days per week & 1.22 & $0.97-1.54$ & 1.18 & $0.90-1.54$ & 1.36 & $0.87-2.11$ \\
\hline $1-3$ days per month & 1.15 & $0.87-1.51$ & 1.05 & $0.76-1.43$ & 1.53 & $0.88-2.65$ \\
\hline Almost never & 0.92 & $0.65-1.29$ & 0.86 & $0.59-1.26$ & 1.16 & $0.55-2.45$ \\
\hline Employment change (Reference: No) & & & & & & \\
\hline Yes & 1.11 & $0.92-1.34$ & 1.17 & $0.94-1.45$ & 0.94 & $0.65-1.36$ \\
\hline Family finances (Reference: Average) & & & & & & \\
\hline Severe & 1.80 & $1.42-2.30$ & 1.94 & $1.47-2.56$ & 1.34 & $0.79-2.26$ \\
\hline Below average & 1.25 & $1.02-1.53$ & 1.19 & $0.94-1.52$ & 1.35 & $0.91-2.02$ \\
\hline Not severe & 0.20 & $0.07-0.55$ & 0.14 & $0.03-0.55$ & 0.40 & $0.10-1.67$ \\
\hline Psychological distress (Reference: $\mathrm{K} 6<$ & & & & & & \\
\hline $\mathrm{K} 6 \geq 13$ & 1.19 & $0.87-1.64$ & 1.16 & $0.78-1.70$ & 1.19 & $0.67-2.10$ \\
\hline Social network (Reference: LSN_6 $\geq 12$ ) & & & & & & \\
\hline LSN_6 $<12$ & 0.89 & $0.74-1.08$ & 0.91 & $0.73-1.14$ & 0.87 & $0.60-1.26$ \\
\hline Alcohol consumption (Reference: $<4$ dri & & & & & & \\
\hline$\geq 4$ drinks & 2.29 & $1.86-2.81$ & 2.10 & $1.68-2.61$ & 3.94 & $2.26-6.85$ \\
\hline
\end{tabular}

CAGE, cutting down, annoyed by criticism, guilty feeling, and eye-opener questionnaire; LSN, Lubben social network scale; PCL, PTSD checklist-specific; K6, Kessler psychological distress scale. 
Table 5. Multivariate analysis: possible risk factors for problem drinking according to increase in CAGE score (PCL only).

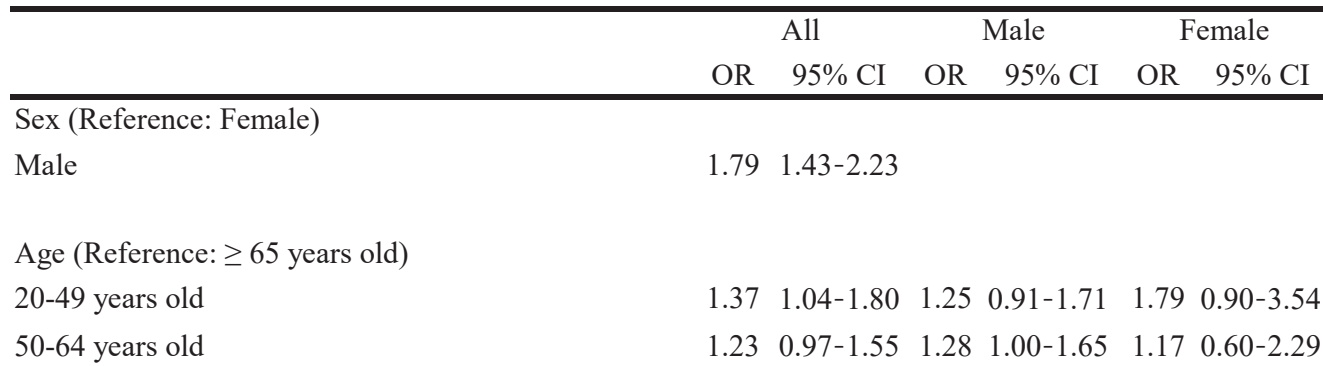

Subjective health condition (Reference: Very good-Good)

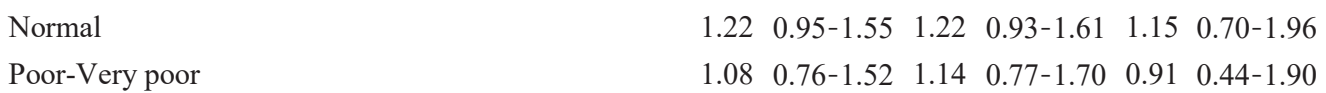

Diagnosed history of mental illness (Reference: None)

Yes

$\begin{array}{llllll}1.23 & 0.85-1.77 & 0.95 & 0.60-1.51 & 2.15 & 1.18-3.94\end{array}$

Diagnosed with high blood pressure (Reference: No)

Yes

$1.11 \quad 0.90-1.38 \quad 1.14 \quad 0.90-1.44 \quad 0.99 \quad 0.60-1.62$

Diagnosed with diabetes mellitus (Reference: No)

Yes

$\begin{array}{llllll}1.15 & 0.91-1.47 & 1.18 & 0.91-1.52 & 0.96 & 0.46-2.00\end{array}$

Sleep insufficiency (Reference: Satisfied)

A little dissatisfied

$\begin{array}{llllll}1.33 & 1.08-1.65 & 1.20 & 0.94-1.52 & 2.24 & 1.32-3.82\end{array}$

Very dissatisfied to quite problematic

$\begin{array}{llllll}1.57 & 1.17-2.10 & 1.34 & 0.95-1.89 & 2.92 & 1.57-5.42\end{array}$

Laughing frequency (Reference: Almost every day)

1-5 days per week

$\begin{array}{llllll}1.24 & 0.98-1.57 & 1.22 & 0.92-1.61 & 1.32 & 0.84-2.07\end{array}$

1-3 days per month

$\begin{array}{lllllll}1.23 & 0.94-1.63 & 1.14 & 0.83-1.58 & 1.66 & 0.95-2.89\end{array}$

Almost never

$\begin{array}{llllll}0.92 & 0.65-1.29 & 0.88 & 0.60-1.30 & 1.01 & 0.45-2.27\end{array}$

Employment change (Reference: No)

Yes

$\begin{array}{llllll}1.08 & 0.89-1.30 & 1.12 & 0.90-1.39 & 0.96 & 0.66-1.41\end{array}$

Family finances (Reference: Average)

Severe

$\begin{array}{llllll}1.70 & 1.33-2.19 & 1.79 & 1.35-2.38 & 0.93 & 0.55-1.58\end{array}$

Below average

$\begin{array}{lllllll}1.21 & 0.98-1.49 & 1.16 & 0.91-1.49 & 0.74 & 0.74-1.27\end{array}$

Not severe

$\begin{array}{llllll}0.21 & 0.08-0.58 & 0.15 & 0.04-0.59 & 0.31 & 0.07-1.36\end{array}$

Trauma (Reference: PCL $<44$ )

$\mathrm{PCL} \geq 44$

$\begin{array}{lllllll}1.77 & 1.38-2.27 & 2.05 & 1.54-2.74 & 1.14 & 0.68-1.92\end{array}$

Social network (Reference: LSN_6 $\geq 12$ )

LSN $6<12$

$\begin{array}{llllll}0.83 & 0.68-1.00 & 0.85 & 0.68-1.07 & 0.80 & 0.54-1.17\end{array}$

Alcohol consumption (Reference: $<4$ drinks)

$\geq 4$ drinks

$\begin{array}{llllll}2.23 & 1.81-2.76 & 2.06 & 1.65-2.58 & 3.78 & 2.08-6.86\end{array}$

CAGE, cutting down, annoyed by criticism, guilty feeling, and eye-opener questionnaire; LSN, Lubben social network scale; PCL, PTSD checklist-specific; K6, Kessler psychological distress scale. 
tims from 2002-2004 and noted that men were more likely than women to begin drinking during stressful times during the second to the fourth year after the disaster. Accordingly, men may be at greater risk of developing problem drinking after traumatic events compared to women.

There are several potential reasons for the sex differences observed. Jayawickreme et al. (2012) examined sex differences in association with trauma cognition and alcohol cravings among individuals diagnosed with PTSD and alcohol dependence and found that negative beliefs about the world and oneself related to traumatic experiences explained alcohol problems in men, while interpersonal relationship problems were a better explanation of problem drinking in women. Therefore, the fact that trauma symptoms (PCL-S) were stronger predictors of problemdrinking development in men might be the result of negative cognitions related to traumatic experiences; conversely, the risk factors among women might be due to the symptoms of an existing illness resulting from longterm evacuation.

The necessity of having to accommodate to a new living style due to long-term evacuation might therefore be inferred as a cause of deterioration in mental functioning in women. Matsumoto et al. (2011) reported that women with problem drinking had severe depression and exhibited greater suicidality than men. Alcohol and depression are interdependent in women and exert a strong influence on each other compared to their effects in men. We presented key data on the risk factors of problem-drinking development among Fukushima evacuees following the Great East Japan Earthquake. The prevalence of CAGE scores $\geq 2$ in this study after adjusting for the age and sex distribution of Japan in 2002 was $10.6 \%$, which is higher than the $3.8 \%$ reported by a nationwide survey in 2002 (Osaki et al. 2005). Thus, the prevalence of problem drinking in the post-disaster period was roughly three times larger than that under non-disaster conditions. However, it should be noted that there may be differences in other risk factors for problem drinking (e.g., survey year, economic status), in addition to controlling for age and sex.

To the best of our knowledge, this is the first study to examine the risk factors of problem drinking in both sexes using the CAGE questionnaire based on a prospective study after the Great East Japan Earthquake. The findings emphasize that problem drinking in the post-disaster period is not a personal issue but a social one. Developing social facilities in evacuation areas that provide physical, psychological, social, and economical support for evacuees is crucial to prevent problem drinking after a disaster. Since sleep insufficiency, history of a diagnosed mental illness, trauma symptoms, and heavy drinking were associated with the development of problem drinking from the second to the third year after the disaster, it is important for practitioners to assess what evacuees need to alleviate mental illness, trauma symptoms, and sleeping issues as a primary measure for the prevention of problem drinking.
Additionally, we found that employment change due to disasters is associated with risk factors for problem drinking in men. Therefore, the government should consider methods to better stabilize occupational situations and economic issues in evacuation areas to support evacuees.

On the other hand, the present study has several limitations. First, the response rate was $19.9 \%$ in 2012; therefore, the results might not represent the wider population of evacuees within the evaluation areas specified by the government. Further, we have excluded participants who failed to answer the CAGE or did not respond to the questionnaire personally. Respondents who completed the CAGE in 2012 reported better status in terms of problem drinking risk factors, except alcohol consumption (e.g., sleep insufficiency, family finance, trauma symptom), than those who responded in 2012, but were excluded due to missing CAGE data or failure to respond on their own (Table 6). This suggested that the prevalence and incidence of problem drinking obtained in this study might be an underestimate of the true incidence rate following the disaster. In this regard, however, bivariate logistic regression analysis showed no large differences in odds ratios for prevalence of problem drinking except for sex and family finances between those included in the data analysis and those excluded due to not responding to the survey in 2013 (Table 7). The associations of problem drinking with sex and family finances were weaker and stronger in those included in this study than those excluded, respectively. Even though the response rate was only $29.9 \%$ in 2012 , this study has gleaned important perspectives of the evacuees residing in the designated evacuation area from the second to third year after the Great East Japan Earthquake on what risk factors led them to develop problem drinking behavior.

Second, we assessed participants' problem drinking using the CAGE questionnaire, which is not equivalent to a clinical diagnosis. Still, the CAGE questionnaire has proven useful as a screening test for alcoholism (Ewing 1984). Third, we did not evaluate the impact of disaster on problem drinking immediately after the disaster because of the lack of the pre-earthquake advent data for this cohort study; however, our study evaluated the risk factors of problem dinking accounting for sex differences in the chronic phase after the compound disaster.

In conclusion, this study has suggested that the consequences of long-term evacuation and the resulting physical, psychological, and economic issues contribute to the development of problem drinking from the second to the third-year post-disaster. The findings of this study may be of use for future disaster preparedness and responses, particularly in terms of policy planning and interventions for evacuees. The government should consider these risk factors in order to provide support and improve evacuees' health and lives after the disaster. 
Table 6. Comparison of socio-demographic, health-related, and disaster-related status in 2012 between individuals whose data were analyzed and those who were excluded.

\begin{tabular}{|c|c|c|c|c|c|c|c|}
\hline & \multirow{2}{*}{$\begin{array}{c}\text { Those who answered } \\
\text { CAGE in } 2012 \\
(36,693) \\
\text { n }\end{array}$} & \multicolumn{3}{|c|}{$\begin{array}{l}\text { Those who } \\
\text { responded in } 2012 \\
\text { but were excluded } \\
\qquad(15,909)\end{array}$} & \multirow[b]{2}{*}{ df } & \multirow[b]{2}{*}{$\chi^{2}$} & \multirow[b]{2}{*}{$p$} \\
\hline & & $\%$ & $\mathrm{n}$ & $\%$ & & & \\
\hline \multicolumn{8}{|l|}{$\overline{\operatorname{Sex}}$} \\
\hline Male & 17,788 & 48.5 & 5,428 & 34.1 & 1 & 928.0 & $<0.001$ \\
\hline Female & 18,905 & 51.5 & 10,481 & 65.9 & & & \\
\hline \multicolumn{8}{|l|}{ Age } \\
\hline 20-49 years old & 12,553 & 34.2 & 1,631 & 10.3 & & & \\
\hline $50-64$ years old & 12,285 & 33.5 & 2,927 & 18.4 & 2 & 7069.5 & $<0.001$ \\
\hline$\geq 65$ years old & 11,855 & 32.3 & 11,351 & 71.3 & & & \\
\hline \multicolumn{8}{|l|}{ Subjective health condition } \\
\hline Very good-Good & 7,383 & 20.6 & 1,707 & 11.4 & & & \\
\hline Normal & 22,515 & 62.9 & 9,028 & 60.5 & 2 & 1220.3 & $<0.001$ \\
\hline Poor-Very poor & 5,875 & 16.4 & 4180 & 28.0 & & & \\
\hline \multicolumn{8}{|l|}{ Diagnosed history of mental illness } \\
\hline No & 33,207 & 92.3 & 12,576 & 86.1 & 1 & 464.4 & $<0.001$ \\
\hline Yes & 2,760 & 7.7 & 2,024 & 13.9 & & & \\
\hline \multicolumn{8}{|l|}{ Sleep insufficiency } \\
\hline Satisfied & 13,054 & 36.6 & 6,140 & 41.9 & & & \\
\hline A little dissatisfied & 16,626 & 46.6 & 6,002 & 41.0 & 2 & 151.1 & $<0.001$ \\
\hline ry dissatisfied to quite problematic & 5,979 & 16.8 & 2,506 & 17.1 & & & \\
\hline \multicolumn{8}{|l|}{ Laughing frequency } \\
\hline Almost every day & 10,059 & 27.6 & 3,883 & 25.3 & & & \\
\hline 1-5 days per week & 14,981 & 41.1 & 5,956 & 38.9 & 3 & 143.8 & $<0.001$ \\
\hline 1-3 days per month & 7,045 & 19.3 & 3,103 & 20.3 & & & \\
\hline Almost never & 4,361 & 12.0 & 2,380 & 15.5 & & & \\
\hline \multicolumn{8}{|l|}{ Socioeconomic factors } \\
\hline \multicolumn{8}{|l|}{ Employment change } \\
\hline Yes & 17,756 & 52.7 & 4,931 & 44.7 & 1 & 217.1 & $<0.001$ \\
\hline No & 15,917 & 47.3 & 6,112 & 55.3 & & & \\
\hline \multicolumn{8}{|l|}{ Family finances } \\
\hline Severe & 5,648 & 16.3 & 2,438 & 18.6 & & & \\
\hline Below average & 10,942 & 31.6 & 3,949 & 30.2 & 3 & 44.8 & $<0.001$ \\
\hline Average & 16,879 & 48.7 & 6,311 & 48.2 & & & \\
\hline Not severe & 1,196 & 3.5 & 383 & 2.9 & & & \\
\hline \multicolumn{8}{|l|}{ Psychological distress } \\
\hline $\mathrm{K} 6<13$ & 31,536 & 89.4 & 10,692 & 83.2 & 1 & 339.0 & $<0.001$ \\
\hline $\mathrm{K} 6 \geq 13$ & 3,745 & 10.6 & 2,165 & 16.8 & & & \\
\hline \multicolumn{8}{|l|}{ Trauma symptom } \\
\hline PCL $<44$ & 28,527 & 84.7 & 8,810 & 75.2 & 1 & 536.8 & $<0.001$ \\
\hline $\mathrm{PCL} \geq 44$ & 5,147 & 15.3 & 2,902 & 24.8 & & & \\
\hline \multicolumn{8}{|l|}{ Social network } \\
\hline $\begin{array}{l}\text { LSN_6 }<12 \\
\text { LSN_6 } 2 \geq 12\end{array}$ & $\begin{array}{l}14,101 \\
21,216\end{array}$ & $\begin{array}{l}39.9 \\
60.1\end{array}$ & $\begin{array}{l}5,173 \\
8,211\end{array}$ & $\begin{array}{l}38.7 \\
61.3\end{array}$ & 1 & 6.612 & 0.010 \\
\hline \multicolumn{8}{|l|}{ Alcohol consumption (drinks) } \\
\hline$<4$ & 16,427 & 83.8 & 2,809 & 87.9 & 1 & 35.2 & $<0.001$ \\
\hline$\geq 4$ & 3,174 & 16.2 & 386 & 12.1 & & & \\
\hline
\end{tabular}

CAGE, cutting down, annoyed by criticism, guilty feeling, and eye-opener questionnaire; LSN, Lubben social network scale; PCL, PTSD checklist-specific; K6, Kessler psychological distress scale. 
Table 7. Comparison of odds ratios for the prevalence of problem drinking in 2012 between individuals whose data were analyzed and those who were excluded.

\begin{tabular}{|c|c|c|c|c|}
\hline & \multicolumn{2}{|c|}{$\begin{array}{c}\text { Data analysis } 2012 \\
(12,490)\end{array}$} & \multicolumn{2}{|c|}{$\begin{array}{l}\text { Excluded } \\
(13,946)\end{array}$} \\
\hline & OR & $95 \% \mathrm{CI}$ & OR & $95 \% \mathrm{CI}$ \\
\hline \multicolumn{5}{|l|}{ Sex (Reference: Female) } \\
\hline Male & 2.64 & $2.24-3.12$ & 5.35 & $4.60-6.23$ \\
\hline \multicolumn{5}{|l|}{ Age (Reference: $\geq 65$ years old) } \\
\hline $20-49$ years old & 1.08 & $0.90-1.29$ & 0.71 & $0.60-0.84$ \\
\hline $50-64$ years old & 1.15 & $0.98-1.35$ & 0.91 & $0.77-1.07$ \\
\hline \multicolumn{5}{|c|}{ Subjective health condition (Reference: Very good-Good) } \\
\hline Normal & 1.44 & $1.19-1.73$ & 1.22 & $0.98-1.50$ \\
\hline Poor-Very poor & 2.05 & $1.62-2.58$ & 1.77 & $1.39-2.23$ \\
\hline \multicolumn{5}{|l|}{ Exercise (Reference: Every day) } \\
\hline 2-4 times a week & 0.97 & $0.78-1.22$ & 0.91 & $0.71-1.15$ \\
\hline Once a week & 0.84 & $0.65-1.08$ & 1.04 & $0.80-1.35$ \\
\hline None & 0.98 & $0.79-1.21$ & 1.10 & $0.89-1.36$ \\
\hline \multicolumn{5}{|c|}{ Diagnosed history of mental illness (Reference: None) } \\
\hline Yes & 1.79 & $1.40-2.28$ & 1.75 & $1.43-2.15$ \\
\hline \multicolumn{5}{|c|}{ Diagnosed with high blood pressure (Reference: None) } \\
\hline Yes & 1.38 & $1.17-1.61$ & 1.44 & $1.23-1.68$ \\
\hline \multicolumn{5}{|c|}{ Diagnosed with diabetes mellitus (Reference: No) } \\
\hline Yes & 1.26 & $1.06-1.49$ & 1.42 & $1.21-1.67$ \\
\hline \multicolumn{5}{|c|}{ Diagnosed with hyperlipidemia (Reference: No) } \\
\hline Yes & 1.02 & $0.89-1.19$ & 1.10 & $0.95-1.28$ \\
\hline \multicolumn{5}{|l|}{ Sleep insufficiency (Reference: Satisfied) } \\
\hline A little dissatisfied & 1.33 & $1.13-1.56$ & 1.15 & $0.98-1.36$ \\
\hline Very dissatisfied to quite problematic & 1.94 & $1.59-2.37$ & 1.70 & $1.40-2.07$ \\
\hline \multicolumn{5}{|c|}{ Laughing frequency (Reference: Almost every day) } \\
\hline 1-5 days per week & 1.17 & $0.98-1.40$ & 1.24 & $1.02-1.50$ \\
\hline 1-3 days per month & 1.27 & $1.03-1.56$ & 1.42 & $1.15-1.76$ \\
\hline Almost never & 1.38 & $1.08-1.74$ & 1.45 & $1.16-1.83$ \\
\hline \multicolumn{5}{|l|}{ Employment change (Reference: No) } \\
\hline Yes & 1.37 & $1.18-1.59$ & 1.23 & $1.05-1.44$ \\
\hline \multicolumn{5}{|l|}{ Family finances (Reference: Average) } \\
\hline Severe & 2.19 & $1.83-2.63$ & 1.58 & $1.30-1.92$ \\
\hline Below average & 1.36 & $1.15-1.61$ & 1.31 & $1.11-1.55$ \\
\hline Not severe & 0.22 & $0.10-0.49$ & 0.88 & $0.56-1.39$ \\
\hline \multicolumn{5}{|l|}{ Psychological distress (Reference: K6 < 13) } \\
\hline$K 6 \geq 13$ & 1.96 & $1.58-2.44$ & 2.03 & $1.68-2.44$ \\
\hline \multicolumn{5}{|l|}{ Trauma symptoma (Reference: PCL < 44) } \\
\hline $\mathrm{PCL} \geq 44$ & 2.51 & $2.11-3.00$ & 2.22 & $1.87-2.64$ \\
\hline \multicolumn{5}{|l|}{ Social network (Reference: LSN_6 $\geq 12$ ) } \\
\hline LSN_6 $<12$ & 1.15 & $1.00-1.33$ & 1.08 & $0.93-1.24$ \\
\hline Alcohol consumption (Reference: $<4 \mathrm{dr}$ & & & & \\
\hline$\geq 4$ drinks & 2.21 & $1.85-2.64$ & 2.92 & $2.30-3.70$ \\
\hline
\end{tabular}

CAGE, cutting down, annoyed by criticism, guilty feeling, and eye-opener questionnaire; LSN, Lubben social network scale; PCL, PTSD checklist-specific; K6, Kessler psychological distress scale. 


\section{Acknowledgments}

We thank the chairpersons, other expert committee members, advisors, and staff of the Fukushima Health Survey Group for conducting this survey and for their support. The findings and conclusions of this article are solely the responsibility of the authors and do not represent the official views of the Fukushima Prefecture government.

This survey was conducted as part of Fukushima Prefecture's post-disaster recovery plans and was supported by the national "Health Fund for Children and Adults Affected by the Nuclear Incident."

\section{Author Contributions}

Y.U., M.M., H.Y., and T.O. conceived and designed the study, collected the data, analyzed and interpreted the data, and drafted the manuscript. M.M., Y.S., M.O., M.M., and S.Y. conceived and designed the study, analyzed and interpreted the data, and critically revised the manuscript. All authors read and approved the final manuscript.

\section{Conflict of Interest}

The authors declare no conflict of interest. The founding sponsor had a role in the design of the study and in the collection of the data.

\section{References}

Ahern, J. \& Galea, S. (2006) Social context and depression after a disaster: the role of income inequality. J. Epidemiol. Community Health, 60, 766-770.

Bell, S., Britton, A., Kubinova, R., Malyutina, S., Pajak, A. \& Nikitin,Y. (2014) Drinking pattern, abstention and problem drinking as risk factors for depressive symptoms: evidence from three urban Eastern European populations. PLoS One, 9, e104384.

Blanchard, E.B., Jones-Alexander, J., Buckley, T.C. \& Forneris, C.A. (1996) Psychometric properties of the PTSD Checklist (PCL). Behav. Res. Ther., 34, 669-673.

Boscarino, J.A., Adams, R.E. \& Galea, S. (2006) Alcohol use in New York after the terrorist attacks: a study of the effects of psychological trauma on drinking behavior. Addict. Behav., 31, 606-621.

Boscarino, J.A., Kirchner, H.L., Hoffman, S.N., Sartorius, J. \& Adams, R.E. (2011) PTSD and alcohol use after the World Trade Center attacks: a longitudinal study. J. Trauma. Stress, 24, 515-525.

Castells, M.A. \& Furlanetto, L.M. (2005) Validity of the CAGE questionnaire for screening alcohol-dependent inpatients on hospital wards. Braz. J. Psychiatry, 27, 54-57.

Cerda, M., Tracy, M. \& Galea, S. (2011) A prospective population based study of changes in alcohol use and binge drinking after a mass traumatic event. Drug Alcohol Depend., 115, 1-8.

Cerda, M., Vlahov, D., Tracy, M. \& Galea, S. (2008) Alcohol use trajectories among adults in an urban area after a disaster: evidence from a population-based cohort study. Addiction, 103, 1296-1307.

Ewing, J.A. (1984) Detecting alcoholism. The CAGE questionnaire. JAMA, 252, 1905-1907.

Fullerton, C.S., McKibben, J.B., Reissman, D.B., Scharf, T., Kowalski-Trakofler, K.M., Shultz, J.M. \& Ursano, R.J. (2013) Posttraumatic stress disorder, depression, and alcohol and tobacco use in public health workers after the 2004 Florida hurricanes. Disaster Med. Public Health Prep., 7, 89-95.

Furukawa, T.A., Kawakami, N., Saitoh, M., Ono, Y., Nakane, Y., Nakamura, Y., Tachimori, H., Iwata, N., Uda, H., Nakane, H., Watanabe, M., Naganuma, Y., Hata, Y., Kobayashi, M.,
Miyake, Y., et al. (2008) The performance of the Japanese version of the K6 and K10 in the World Mental Health Survey Japan. Int. J. Methods Psychiatr. Res., 17, 152-158.

Gray, J., Kim, J., Ciesla, J.R. \& Yao, P. (2016) Rasch analysis of the Lubben Social Network Scale-6 (LSNS-6). J. Appl. Gerontol., 35, 508-528.

Hasan, H. \& Hasan, T.F. (2009) Laugh yourself into a healthier person: a cross cultural analysis of the effects of varying levels of laughter on health. Int. J. Med. Sci., 6, 200-211.

Hayashi, K., Kawachi, I., Ohira, T., Kondo, K., Shirai, K. \& Kondo, N. (2015) Laughter and subjective health among community-dwelling older people in Japan: cross-sectional analysis of the Japan gerontological evaluation study cohort data. J. Nerv. Ment. Dis., 203, 934-942.

Hirosaki, M., Ohira, T., Yasumura, S., Maeda, M., Yabe, H., Harigane, M., Takahashi, H., Murakami, M., Suzuki, Y., Nakano, H., Zhang, W., Uemura, M., Abe, M. \& Kamiya, K.; Fukushima Health Management Survey Group (2018) Lifestyle factors and social ties associated with the frequency of laughter after the Great East Japan Earthquake: Fukushima Health Management Survey. Qual. Life Res., 27, 639-650.

Iwasa, H., Suzuki, Y., Shiga, T., Maeda, M., Yabe, H. \& Yasumura, S.; Mental Health Group of the Fukushima Health Management Survey (2016) Psychometric evaluation of the Japanese version of the posttraumatic stress disorder checklist in community dwellers following the Fukushima Daiichi Nuclear Power Plant Incident: the Fukushima Health Management Survey. SAGE Open, 6, doi.org/10.1177/2158244016652444.

Jayawickreme, N., Yasinski, C., Williams, M. \& Foa, E.B. (2012) Gender-specific associations between trauma cognitions, alcohol cravings, and alcohol-related consequences in individuals with comorbid PTSD and alcohol dependence. Psychol. Addict. Behav., 26, 13-19.

Kachadourian, L.K., Pilver, C.E. \& Potenza, M.N. (2014) Trauma, PTSD, and binge and hazardous drinking among women and men: findings from a national study. J. Psychiatr. Res., 55, $35-43$.

Karz, A., Reichstein, J., Yanagisawa, R. \& Katz, C.L. (2014) Ongoing mental health concerns in post-3/11 Japan. Ann. Glob. Health, 80, 108-114.

Kessler, R.C., Barker, P.R., Colpe, L.J., Epstein, J.F., Gfroerer, J.C., Hiripi, E., Howes, M.J., Normand, S.L., Manderscheid, R.W., Walters, E.E. \& Zaslavsky, A.M. (2003) Screening for serious mental illness in the general population. Arch. Gen. Psychiatry, 60, 184-189.

Kessler, R.C., Galea, S., Jones, R.T. \& Parker, H.A.; Hurricane Katrina Community Advisory Group (2006) Mental illness and suicidality after Hurricane Katrina. Bull. World Health Organ., 84, 930-939.

Kurimoto, A., Awata, S., Ohkubo, T., Tsubota-Utsugi, M., Asayama, K., Takahashi, K., Suenaga, K., Satoh, H. \& Imai, Y. (2011) Reliability and validity of the Japanese version of the abbreviated Lubben Social Network Scale. Nihon Ronen Igakkai Zasshi, 48, 149-157 (in Japanese).

Lavie, P. (2001) Sleep disturbances in the wake of traumatic events. N. Engl. J. Med., 345, 1825-1832.

Lubben, J., Blozik, E., Gillmann, G., Iliffe, S., von Renteln Kruse, W., Beck, J.C. \& Stuck, A.E. (2006) Performance of an abbreviated version of the Lubben Social Network Scale among three European community-dwelling older adult populations. Gerontologist, 46, 503-513.

Matsumoto, S., Yamaoka, K., Inoue, M. \& Muto, S.; Teikyo Ishinomaki Research Group and Health and Life Revival Council in the Ishinomaki district (RCI) (2014) Social ties may play a critical role in mitigating sleep difficulties in disaster-affected communities: a cross-sectional study in the Ishinomaki area, Japan. Sleep, 37, 137-145.

Matsumoto, T., Azekawa, T., Uchikado, H., Ozaki, S., Hasegawa, N., Takekawa, Y. \& Matsushita, S. (2011) Comparative study 
of suicide risk in depressive disorder patients with and without problem drinking. Psychiatry Clin. Neurosci., 65, 529-532.

Ministry of Health, Labour and Welfare (2019) Alcohol. https://www.mhlw.go.jp/www1/topics/kenko21_11/b5.html [Accessed: May 25, 2019] (in Japanese).

Nordløkken, A., Pape, H. \& Heir, T. (2016) Alcohol consumption in the aftermath of a natural disaster: a longitudinal study. Public Health, 132, 33-39.

North, C.S., Ringwalt, C.L., Downs, D., Derzon, J. \& Galvin, D. (2011) Postdisaster course of alcohol use disorders in systematically studied survivors of 10 disasters. Arch. Gen. Psychiatry, 68, 173-180.

Oe, M., Fujii, S., Maeda, M., Nagai, M., Harigane, M., Miura, I., Yabe, H., Ohira, T., Takahashi, H., Suzuki, Y., Yasumura, S. \& Abe, M. (2016) Three-year trend survey of psychological distress, post-traumatic stress, and problem drinking among residents in the evacuation zone after the Fukushima Daiichi Nuclear Power Plant accident [The Fukushima Health Management Survey]. Psychiatry Clin. Neurosci., 70, 245-252.

Orui, M., Ueda, Y., Suzuki, Y., Maeda, M., Ohira, T., Yabe, H. \& Yasumura, S. (2017) The relationship between starting to drink and psychological distress, sleep disturbance after the Great East Japan Earthquake and nuclear disaster: the Fukushima Health Management Survey. Int. J. Environ. Res. Public Health, 14, 1281.

Osaki, Y., Matsushita, S., Shirasaka, T., Hiro, H. \& Higuchi, S. (2005) Nationwide survey of alcohol drinking and alcoholism among Japanese adults. Nihon Arukoru Yakubutsu Igakkai Zasshi, 40, 455-470 (in Japanese).

Petrakis, I.L. \& Simpson, T.L. (2017) Posttraumatic stress disorder and alcohol use disorder: a critical review of pharmacologic treatments. Alcohol. Clin. Exp. Res., 41, 226-237.

Suzuki, Y., Yabe, H., Horikoshi, N., Yasumura, S., Kawakami, N., Ohtsuru, A., Mashiko, H. \& Maeda, M.; Mental Health Group of the Fukushima Health Management Survey (2017) Diagnostic accuracy of Japanese posttraumatic stress measures after a complex disaster: the Fukushima Health Management Survey. Asia Pac. Psychiatry, 9, doi: 10.1111/appy.12248. [Epub ahead of print].

Ueda, Y., Yabe, H., Maeda, M., Ohira, T., Fujii, S., Niwa, S., Ohtsuru, A., Mashiko, H., Harigane, M. \& Yasumura, S.; Fukushima Health Management Survey Group (2016) Drinking behavior and mental illness among evacuees in Fukushima following the Great East Japan Earthquake: the Fukushima Health Management Survey. Alcohol. Clin. Exp. Res., 40, 623-630.

Verplaetse, T.L., Moore, K.E., Pittman, B.P., Roberts, W., Oberleitner, L.M., Smith, P.H., Cosgrove, K.P. \& McKee, S.A. (2018) Intersection of stress and gender in association with transitions in past year DSM-5 substance use disorder diagnoses in the United States. Chronic Stress (Thousand Oaks), 2, doi: 10.1177/2470547017752637. [Epub ahead of print].

Vetter, S., Rossegger, A., Rossler, W., Bisson, J.I. \& Endrass, J. (2008) Exposure to the tsunami disaster, PTSD symptoms and increased substance use: an Internet based survey of male and female residents of Switzerland. BMC Public Health, 8, 92.

Vlahov, D., Galea, S., Resnick, H., Ahern, J., Boscarino, J.A., Bucuvalas, M., Gold, J. \& Kilpatrick, D. (2002) Increased use of cigarettes, alcohol, and marijuana among Manhattan, New York, residents after the September 11th terrorist attacks. Am. J. Epidemiol., 155, 988-996.

Welch, A.E., Caramanica, K., Maslow, C.B., Cone, J.E., Farfel, M.R., Keyes, K.M., Stellman, S.D. \& Hasin, D.S. (2014) Frequent binge drinking five to six years after exposure to 9/11: findings from the World Trade Center Health Registry. Drug Alcohol Depend., 140, 1-7.

Yasumura, S., Hosoya, M., Yamashita, S., Kamiya, K., Abe, M., Akashi, M., Kodama, K. \& Ozasa, K.; Fukushima Health Management Survey Group (2012) Study protocol for the Fukushima Health Management Survey. J. Epidemiol., 22, 375-383. 